\title{
The Training Process Optimization Research of Graduate Students in Business Administration based on Information Exchange Platform
}

\author{
Jian $\mathrm{Hu}^{1, \mathrm{a}^{*}}$, Jin Hua Sun ${ }^{1, \mathrm{~b}}$, Mi Mi Yang ${ }^{1, \mathrm{c}}$ and Hui Fang Jian ${ }^{1, \mathrm{~d}}$ \\ ${ }^{1}$ School of Management, Chongqing University of Technology, Chongqing, 400054, China \\ ajianhu-hit@163.com, bsjh1009@163.com, c1401431684@qq.com, 731228119@qq.com
}

\begin{abstract}
Keywords: Training process optimization; Graduate students; Business Administration; Information Exchange Platform.
\end{abstract}

\begin{abstract}
According to the current problems of business administration graduate students training, this paper has been intensively studied on the training process of graduate students in business administration from the perspective of information technology. An overall optimal framework of the optimization project of graduate students in business administration was put forward, which include the training process optimization process node, the results of the training process visualization, process tracking mechanism and culture training process interaction mechanisms. On this basis, practice popularization and application of ideas of optimization project are given to improve the clutivation quality of graduate students education.
\end{abstract}

\section{Introduction}

With the rapid development of China's economy, there is a higher quality requirements for business management personnel, and this high demand for traditional business administration graduate training process presents new challenges[1]. In particular, the growing popularity of information technology today, it is key for universities about how to use modern information technology to continuously optimize postgraduate training process management. It is essential for cultivating high-level research talent that meets the current situation development needs. To solve this problem, although there are many scholars have made depth explorations about the postgraduate training process management, and proposed countermeasures [2-4], but the results of these studies don't effectively improve the quality of graduate education in business administration subjects. The main one reason is that the current process training graduate management lack scientific auxiliary support means. We can say that the traditional business administration graduate training process management is difficult to meet the needs of high-quality personnel training[5].

Therefore, to change the current situation, the various constraints in current training model should be break down. Combined with the characteristics of business management training process, and constantly optimize the training process, which has important theoretical and practical significance about effectively improving the business management postgraduate training quality[6,7]. Therefore, according to the existing problems in current business management graduate training process, this paper combined with the experience accumulated in postgraduate training practices, in-depth study optimization approaches and methods of business management disciplines postgraduate training process based on the perspective of information technology.

\section{Current Status of Postgraduate Training Process Management in China}

The Management of Theoretical Teaching Training Process. The section headings are in boldface capital and lowercase letters. Second level headings are typed as part of the succeeding paragraph (like the subsection heading of this paragraph). Through combing the current research, we know that the current research focus more on the front part of graduate training program - teaching management, teaching management process is considered as the basic guarantee to keeping stable teaching order and complete the task of postgraduate training. Most scholars in graduate management education 
think that the training process is teaching management activity, which can ensure the successful completion of postgraduate training process by the use of teaching resources. We mainly depend on the modern information technology to complete the teaching management process of postgraduate, namely four links, which including research directions, developing a learning plan, getting the courses credit and research prepared. Some other scholars analyze the management of teaching process from the angle of training subject and object. They hold the view that we should pay more attention to the students' creation and development capabilities, which is implicit in the teaching process, only by the scientific and standardized management of the teaching process, can we achieve effective convergence between teachers and students. To further strengthen the postgraduate teaching management, the management process about the teaching process theory is based on the majority of university-based postgraduate training steering group of experts on the establishment of graduate students' credits checkpoint[8].

The Management of Practical Teaching Training Process. Through various Chinese universities graduate training practice management of process analysis, the current graduate practical training process management mainly by scientific practice - interim assessment - theses - dissertation proposal - mid-term examination papers - paper review - thesis defense and many other areas constitution. In this process, more reflects in the importance of responsibility system management mode. Currently, the practical aspects of the training process is also dependent on certain information technology means to achieve the training process. But the mentor has not been fully expressed in the process of the role. Especially the cultivation process management practice guidance session has become a point neglected by scholars in practice guide link. And more research is focused on management by objectives. The results of the above-mentioned aspects of the training objectives through information technology platform to show up. It is not conducive to the practical aspects of the whole process of training to achieve a certain extent. And then affecting the overall innovation capability upgrade graduate[9].

\section{The Existing Problems in the Process Management of Graduate Education in China}

A research about the process management of graduate education have been carried out in part of Chinese colleges and universities ,the analysis shows the existence of many problems, mainly reflected in the overlook of the after process of the cultivation of graduate student - the process of scientific research management, and the lack of effective coordination in graduate education process[10]. Embodied in the following aspects:

The Cultivating Method is Single to Engage in Scientific Research. Nowadays, when the Chinese colleges and universities train the graduate student who majored in the industry and commerce to undertake the scientific research, the method is relatively single. And the ' $t$ ask learn a' training mode have been adopted all the time, which is very unfavorable to the cultivation of innovative thinking of graduate students. This training method makes tutors to be the only strong authority in the scientific research the student engaged in, and students become the controlled body and a single passive accept body who lack coordination and interaction in the training process or the form of benign interaction of a learning division, which results in the decrease of students' efficiency of scientific research and the not enough in-depth understanding of knowledge, also, the independent thinking ability and innovation consciousness is difficult to get trained in class.

The Scientific of Practical Process are Not Reflected. Business administration graduate training methods are more focused on the practical aspects of theoretical knowledge transfer, but the students did not understand these theories in practice, specifically how to use, so that the training methods became paper. Too much emphasis on imparting knowledge, ignoring the graduate students' self-learning ability, which make students in a passive position to accept learning. The interaction in graduate training process and the purpose of cultivating innovative talents training cannot be achieved. As a result, the students' personalized, diversified developments were limited. Especially, the science teaching methods were only focused on the theoretical aspects of indoctrination, not interpreted from 
the method itself practicality. As a result, students cannot to learn the scientific method knowledge in context.

The Training Approach of One to One Reduced the Efficiency of Scientific Research. In the majority of graduate training process, mentoring of students is limited to one to one guidance mode, which to some extent will reduce the efficiency of scientific research that the instructors are engaged in. By face to face, online or communication with students one to one exchange and communication, the benefit coverage is limited to a student's own guidance, which is unable to achieve the economies scale of cultivation. Other members of the research team did not play its internal reference function, and the utility of instructors' guidance work has not been fully realized. All of those are adversely affect the efficiency of research work.

Academic Assessment Process Lacked Effective Method. At present, most academic assessment methods are limited to the consideration of the final outcome of an academic, that is the published papers related, but the lack of effective academic writing process monitoring and evaluation. The phenomenon is management by objectives appears very important, training process management is neglected. Important part - Interim quality assessment is basically non-existent in many schools, the actual assessment work a mere formality, did not play its actual function about assessing student's learning and research capabilities progress. This is not only affected the lower graduate output efficiency to a certain extent, but it is difficult to output a high level of academic achievement, and even some acts of academic misconduct.

\section{Optimized Design of Business Management Disciplines Postgraduate Training Process}

According to the problems occurred in the business administration graduate training process, we design an optimal overall framework of the program, shown in Figure 1.

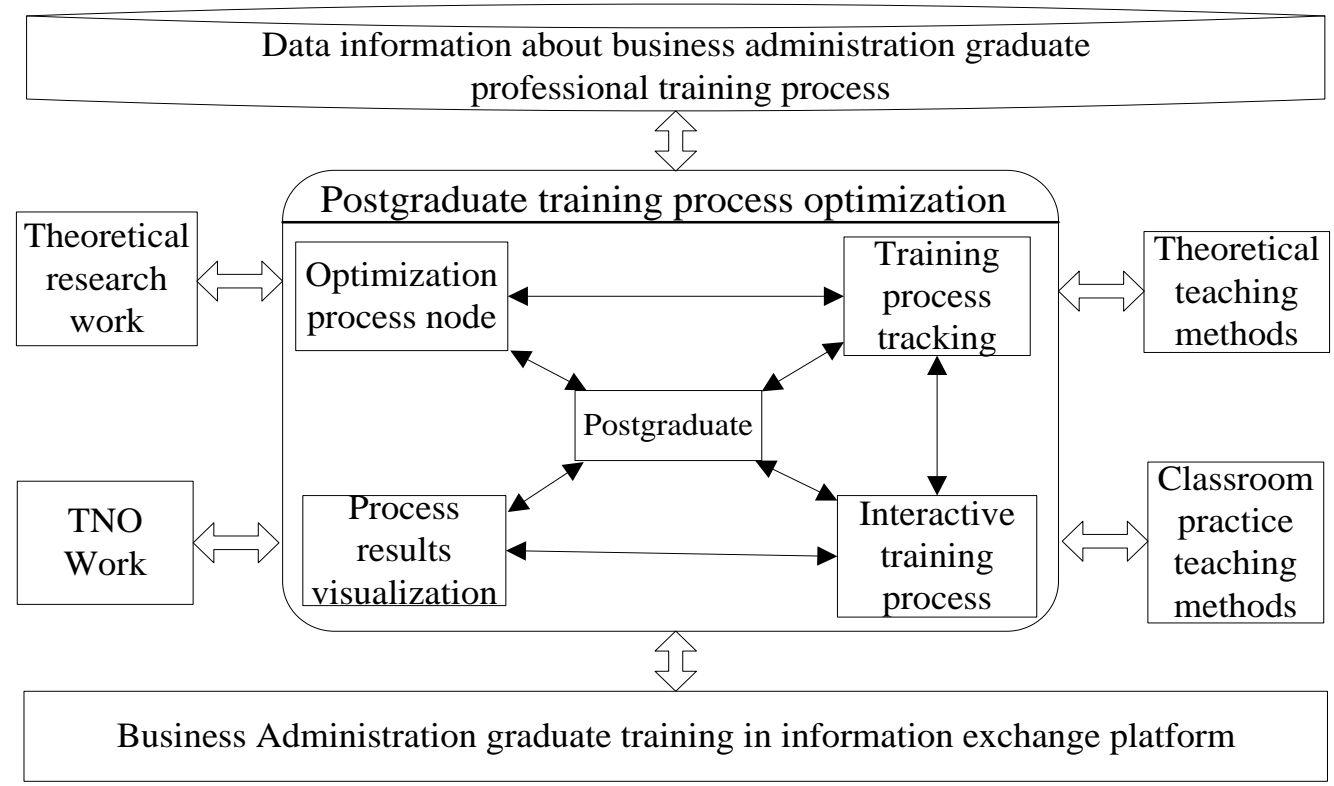

Figure 1. the overall framework of optimal program of business administration graduate training in discipline process

Graduate Training Process Optimization Process Node. For business administration graduate, postgraduate training focused on learning process and characteristics of domestic and foreign universities, summarize the main flow of business administration postgraduate training, and analysis the process to identify key nodes, using flowcharts and other tools to optimize the process node, lay the foundation for the design of information exchange platform.

Graduate training process results visualization. In-depth analysis of Business Administration major work on each node, to explore the outcomes on each node visualization mechanisms and 
pathways. On the basis of the visualization, design the comparison and evaluation mechanism of the graduate outcomes, so students understand their culture problems and deficiencies existing in the process, to further clarify the direction of their own culture.

Graduate training process tracking mechanism. On the basis of the results of the training process optimization and process visualization, designing Graduate training process tracking mechanism. With the information exchange platform to implement the real-time supervision for graduate student, and on this basis, establish an effective graduate appraisal system.

Graduate training process interaction mechanism. With the information exchange platform, establishing interaction mechanisms among graduate students and instructors, graduate and postgraduate, graduate and master tutor other students and teachers and between young Dr. Through interactive training process, providing access to scientific information for graduate students, and a variety of ways and effective ways about research supports.

\section{Practice Popularization and Application of Ideas of Optimization Project}

Choosing a business management major in Business Administration, Chongqing University as a practice target discipline under the program, established specific practice operational processes for business management disciplines postgraduate training process optimization.

The optimization program in the process of graduate training implement the project responsibility in specific training links, taking the project management approach to organizing according to the principle the overall design, step by step.

Security system established to support graduate training in business administration specializing in the process of optimization of program implementation. This system includes both establish the idea of modern teaching philosophy, improve and perfect the mechanism of science policy, and enhance the overall quality of the tutor team and so on.

Develop implementation project management approach of postgraduate training process optimization program and its sub-organizations implement management practices and rules, and to develop appropriate financial management practices and management systems.

\section{Summary}

This article analyzes the current situation of business administration graduate training process in some Chinese universities, founding that there are many problems existing in business administration graduate training process. According to these problems, business administration graduate training professional process optimization was designed. In order to ensure that the optimization scheme can be implemented smoothly, put forward the corresponding practical promotion and application of postgraduate training program for process optimization program. Ultimately, effectively enhance the quality of the graduate management training for industry and commerce.

\section{Acknowledgments}

This work is partially supported by the National Natural Science Foundation of China (Grant No. 71301181), Chongqing City Association of Higher Education Project (No. CQGJ13C436), Chongqing University of Technology Graduate Student Education Reform Project(No.yjg2013209, yjg2014206), Chongqing City Graduate Student Education Reform Project(No.yjg132020)

\section{References}

[1] Yao Jie, Zhu Peijun. Study on the Training Mode of Innovative Graduate Students. Journal of Northeast Dianli University. 5(2012)75-77

[2] Weiwen Huang, Shibo Liu. On the Process Management in Graduate Education Quality Assurance System Applications. Study of Higher Education. 22(2006)47-50 
[3] Dongmei Zheng, Erli Liu. Learn to explore postgraduate training standards established ISO9000 quality management system processes. Degrees and Graduate Education. (2007) 98-100.

[4] Menlou Li. Process management and improve the quality of graduate education. Xiangtan Normal University (Social Sciences). 29(2007) 197-198

[5] Jianhua Hu. Quality of internal management and external monitoring of the relationship between higher education. Higher Education Research. 5(2008)33

[6] Chengdong Qian, Yue Qian, Chunyuan Zhang. Strengthen the training process management, improve the quality of graduate training. Graduate Education.6(2011)41-43

[7] Jin Li, Wan Hongying, Liu Haiqing etal. Initial Applications of a Monitoring and Warning System for the Graduate Training Process. Journal of Graduate Education.3(2011)31-36

[8] Geng You-quan, Peng Wei-na, Peng Zhi-yue etal. Study of Research-Oriented Graduate Training in China-Based on survey data from 14 national key universities. Journal of Graduate Education. 6(2011)28-34

[9] Zhen Liang, Kang Jun, Ying Shuang. Construction of a Quality Evaluation and Assurance System for Professional Master's Degree Programs. Journal of Graduate Education. 6(2012)52-55

[10]Teng Weili. Reflections on the Training of Key Links in the Process of Graduate Students. Journal of Heilongjiang College of Education.32(2013)10-12 